J. Korean Math. Soc. 49 (2012), No. 1, pp. 85-98

http://dx.doi.org/10.4134/JKMS.2012.49.1.085

\title{
THE TOTAL GRAPH OF A COMMUTATIVE RING WITH RESPECT TO PROPER IDEALS
}

\author{
Ahmad Abbasi and Shokoofe Habibi
}

\begin{abstract}
Let $R$ be a commutative ring and $I$ its proper ideal, let $S(I)$ be the set of all elements of $R$ that are not prime to $I$. Here we introduce and study the total graph of a commutative ring $R$ with respect to proper ideal $I$, denoted by $T\left(\Gamma_{I}(R)\right)$. It is the (undirected) graph with all elements of $R$ as vertices, and for distinct $x, y \in R$, the vertices $x$ and $y$ are adjacent if and only if $x+y \in S(I)$. The total graph of a commutative ring, that denoted by $T(\Gamma(R))$, is the graph where the vertices are all elements of $R$ and where there is an undirected edge between two distinct vertices $x$ and $y$ if and only if $x+y \in Z(R)$ which is due to Anderson and Badawi [2]. In the case $I=\{0\}, T\left(\Gamma_{I}(R)\right)=T(\Gamma(R))$; this is an important result on the definition.
\end{abstract}

\section{Introduction}

The concept of total graph of a commutative ring $R$, one of the most interesting concept of the algebraic structures in graph theory denoted by $T(\Gamma(R))$, was first introduced by Anderson and Badawi in [2], such that the vertex set is $R$ and the distinct vertices $x$ and $y$ are adjacent if and only if $x+y \in Z(R)$ where $Z(R)$ is the zero divisors of $R$. Throughout this work all rings are assumed to be commutative with non-zero identity. Let $I$ be a proper ideal of $R$. The total graph of a commutative ring $R$ with respect to proper ideal $I$, denoted by $T\left(\Gamma_{I}(R)\right)$, is the graph which vertices are all elements of $R$ and two distinct vertices $x, y \in R$ are adjacent if and only if $x+y \in S(I)$. We use the notation $S(I)$ to refer to the set of elements of $R$ that are not prime to $I$, we say that $a \in R$ is prime to $I$, if $r a \in I$ (where $r \in R$ ) implies that $r \in I$ (see $[6,7]$ ). Clearly, $S(I)$ is not empty since $I$ is a proper ideal of $R$. It is easy to check that, when $I=\{0\}, T\left(\Gamma_{I}(R)\right)=T(\Gamma(R))$. The zero-divisor graph of $R$, denoted $\Gamma(R)$, is the graph whose vertices are $Z(R)^{*}$ (the nonzero zero-divisors of $R$ ) with two distinct vertices joined by an edge when the product of the vertices is zero (c.f. [3]). In [8], Redmond introduced the zero divisor graph with respect to proper ideal $I$, denoted by $\Gamma_{I}(R)$, as the graph

Received August 24, 2010.

2010 Mathematics Subject Classification. 13D45, 13E10, $13 \mathrm{C} 05$.

Key words and phrases. commutative rings, zero divisor, total graph. 
with vertices $\{x \in R-I: x y \in I$ for some $y \in R-I\}$ where distinct vertices $x$ and $y$ are adjacent if and only if $x y \in I$. If $I=\{0\}$, then $\Gamma_{I}(R)=\Gamma(R)$. Redmond explored the relationship between $\Gamma_{I}(R)$ and $\Gamma(R)$. He gave an example of rings $R, S$ and ideals $I \unlhd R, J \unlhd S$, where $\Gamma(R / I) \cong \Gamma(S / J)$ but $\Gamma_{I}(R) \supsetneqq \Gamma_{J}(S)$. Similarly, in this paper we give an example (see Example 2.2) such that $T\left(\Gamma_{I}(R)\right) \cong T\left(\Gamma_{J}(S)\right)$ but $T(\Gamma(R / I)) \varsubsetneqq T(\Gamma(S / J))$ and some basic results on the relationship between $T\left(\Gamma_{I}(R)\right)$ and $T(\Gamma(R / I))$ in Section 2 .

The set $S(I)$ is not necessarily an ideal of $R$ (not always closed under addition) and since $S(I)$ is a union of prime ideals of $R$ containing $I$ (see [4, Exe. 3.9] and note that 2.1), whenever $x y \in S(I)$ for $x, y \in R$, then $x \in S(I)$ or $y \in S(I)$. So, if $S(I)$ is an ideal of $R$, then it is actually a prime ideal of $R$; hence the study of $T\left(\Gamma_{I}(R)\right)$ breaks naturally into two cases depending on whether or not $S(I)$ is an ideal of $R$ and in Sections 3,4, we state several results about the relationship between diameter and girth of $T\left(\Gamma_{I}(R)\right)$ and $T(\Gamma(R / I))$. The proper ideal $I$ is said to be $P$-primal ideal of $R$ when $P=S(I)$ forms an ideal; then $P$ is said to be the adjoint ideal of $I$. It is easy to see that, $S(I)=I$ when $I$ is a prime ideal $R$ (see $[6,7])$. Let $S\left(\Gamma_{I}(R)\right)$ be the (induced) subgraph of $T\left(\Gamma_{I}(R)\right)$ with vertices $S(I)$, and let $\bar{S}\left(\Gamma_{I}(R)\right)$ be the (induced) subgraph $T\left(\Gamma_{I}(R)\right)$ with vertices $R-S(I)$.

Let $G$ be a graph with vertex set $V(G)$. Recall that $G$ is connected if there is a path between any two distinct vertices of $G$. At the other extreme, we say that $G$ is totally disconnected if no two vertices of $G$ are adjacent. For vertices $x$ and $y$ of $G, d(x, y)$ be the length of a shortest path from $x$ to $y(d(x, x)=0$ and $d(x, y)=\infty$ if there is no such path). The diameter of a graph $G$, denoted by $\operatorname{diam}(G)$, is the supremum of the distances between vertices. The girth of $G$, denoted by $\operatorname{gr}(G)$, is the length of a shortest cycle in $G(\operatorname{gr}(G)=\infty$ if $G$ contains no cycles). A graph $G$ is said to be complete bipartite if $V(G)$ can be partitioned into two disjoint sets $V_{1}, V_{2}$ such that no two vertices within any $V_{1}$ or $V_{2}$ are adjacent, but for every $u \in V_{1}, v \in V_{2}, u, v$ are adjacent. Then we use the symbol $K^{m, n}$ for the complete bipartite graph where the cardinal numbers of $V_{1}$ and $V_{2}$ are $m, n$, respectively (we allow $m$ and $n$ to be infinite cardinals). A graph in which each pair of distinct vertices is joined by an edge is called a complete graph. Let $K_{n}$ denote the complete graph with $n$ vertices.

In Section 2, we obtain an identity between completeness of $\bar{S}\left(\Gamma_{I}(R)\right)$ and $\operatorname{Reg} \Gamma(R / I)$. We study the Graphs $T\left(\Gamma_{I}(R)\right), S\left(\Gamma_{I}(R)\right)$ and $\bar{S}\left(\Gamma_{I}(R)\right)$ for the case when $S(I)$ is an ideal in Section 3 and for the case $S(I)$ is not an ideal in Section 4. Though our definition of total graph of a commutative ring is a generalization of the definition given in [2], we would like to point out that many of the proofs provided in this paper are essentially the same as the proofs provided in $[2]$. 


\section{Example and basic structure}

In this section, we explore the relationship between $T\left(\Gamma_{I}(R)\right)$ and $T(\Gamma(R / I))$ on basic structure.

Note 2.1. We can easily show that $Z(R / I)=\{a+I: a \in S(I)\}$ and $\operatorname{Reg}(R / I)=\{a+I: a \notin S(I)\}$. Thus $Z(R / I)$ is an ideal $R / I$ if and only if $S(I)$ is an ideal $R$.

Let $\operatorname{Reg}(\Gamma(R / I))$ be the (induced) subgraph of $T(\Gamma(R / I))$ with vertices $\operatorname{Reg}(R / I)$, the set of regular elements of $R / I$, let $Z(\Gamma(R / I))$ be the (induced) subgraph of $T(\Gamma(R / I))$ with vertices $Z(R / I)$.

Example 2.2. Let $R=\mathbb{Z}_{8}, S=\mathbb{Z}_{4} \times \mathbb{Z}_{2}$ and $I=\{\overline{0}, \overline{2}, \overline{4}, \overline{6}\} \unlhd R, J=\{\overline{0}\} \times$ $\mathbb{Z}_{2} \unlhd S$. It is easy to check that $S(I)=I$ and $S(J)=\{(\overline{0}, \overline{0}),(\overline{0}, \overline{\overline{1}}),(\overline{2}, \overline{0}),(\overline{2}, \overline{1})\}$. $T\left(\Gamma_{I}(R)\right)$ and $T\left(\Gamma_{J}(S)\right)$ are the union of 2 disjoint $K^{4,} s$. Now, $T(\Gamma(R / I))$ is a graph with two vertices but $T(\Gamma(S / J))$ is a graph with four vertices.

Theorem 2.3. Let $R$ be a commutative ring with the proper ideal $I$, and let $x, y \in R$. Then

(1) If $x+I$ and $y+I$ are (distinct) adjacent vertices in $T(\Gamma(R / I))$, then $x$ is adjacent to $y$ in $T\left(\Gamma_{I}(R)\right)$.

(2) If $x$ and $y$ are (distinct) adjacent vertices in $T\left(\Gamma_{I}(R)\right)$ and $x+I \neq y+I$, then $x+I$ is adjacent to $y+I$ in $T(\Gamma(R / I))$.

(3) If $x$ is adjacent to $y$ in $T\left(\Gamma_{I}(R)\right)$ and $x+I=y+I$, then $2 x, 2 y \in S(I)$ and all distinct elements of $x+I$ are adjacent in $T\left(\Gamma_{I}(R)\right)$.

Proof. It is clear.

According to the following corollary and remark, there is a strong relationship between $T(\Gamma(R / I))$ and $T\left(\Gamma_{I}(R)\right)$.

Note that for a graph $G$, we say that $\left\{G_{\theta}\right\}_{\theta \in \Theta}$ is a collection of disjoint subgraphs of $G$ if all vertices and edges of each $G_{\theta}$ are contained in $G$ and no two of these $G_{\theta}$ contain a common vertex.

Corollary 2.4. Let $R$ be a commutative ring with the proper ideal I. Then $T\left(\Gamma_{I}(R)\right)$ contains $|I|$ disjoint subgraphs isomorphic to $T(\Gamma(R / I))$.

Proof. Let $\left\{a_{\lambda}\right\}_{\lambda \in \Lambda} \subseteq R$ be a set of distinct representatives of the vertices of $T(\Gamma(R / I))$. Define a graph $G_{i}$, for each $i \in I$, with vertices $\left\{a_{\lambda}+i \mid \lambda \in \Lambda\right\}$, where $a_{\lambda}+i$ is adjacent to $a_{\beta}+i$ in $G_{i}$ whenever $a_{\lambda}+I$ is adjacent to $a_{\beta}+I$ in $T(\Gamma(R / I))$; i.e., whenever $a_{\lambda}+a_{\beta} \in S(I)$. By the above theorem, $G_{i}$ is a subgraph of $T\left(\Gamma_{I}(R)\right)$. Also, each $G_{i} \cong T(\Gamma(R / I))$, and $G_{i}$ and $G_{j}$ contains no common vertices if $i \neq j$.

Remark 2.5. It follows from the above corollary that $S\left(\Gamma_{I}(R)\right)$ contains $|I|$ disjoint subgraphs isomorphic to $Z(\Gamma(R / I))$ and $\bar{S}\left(\Gamma_{I}(R)\right)$ contains $|I|$ disjoint subgraphs isomorphic to $\operatorname{Reg}(\Gamma(R / I))$; since for each $a \in S(I)$ and $b \in R-S(I)$, and $i \in I ; a+i \in S(I)$ (for some $r \in R-I$, ar $\in I$; hence $(a+i) r \in I$ ) and 
$b+i \in R-S(I)$. So a graph $G_{i}$ with vertices $\left\{a_{\lambda}+i \mid \lambda \in \Lambda\right\}$ such that $a_{\lambda} \in S(I)$ is a subgraph $S\left(\Gamma_{I}(R)\right)$ and a graph $G_{i}$ with vertices $\left\{a_{\beta}+i \mid \beta \in \Lambda\right\}$ such that $a_{\beta} \notin S(I)$ is a subgraph $\bar{S}\left(\Gamma_{I}(R)\right)$.

One can verify that the following method can be used to construct a graph $T\left(\Gamma_{I}(R)\right)$.

Remark 2.6. Let $\left\{a_{\lambda}\right\}_{\lambda \in \Lambda} \subseteq R$ be a set of representatives of the vertices of $T(\Gamma(R / I))$. For each $i \in I$, define a graph $G_{i}$ with vertices $\left\{a_{\lambda}+i \mid \lambda \in \Lambda\right\}$, where edges are defined by the relationship $a_{\lambda}+i$ is adjacent to $a_{\beta}+i$ in $G_{i}$ if and only if $a_{\lambda}+I$ is adjacent to $a_{\beta}+I$ in $T\left(\Gamma(R / I)\right.$ ) (i.e., $a_{\lambda}+a_{\beta} \in S(I)$ ). Define the graph $G$ to have as its vertex set $V=\bigcup_{i \in I} G_{i}$. We define the edge set of $G$ to be:

(1) all edges contained in $G_{i}$ for each $i \in I$,

(2) for distinct $\lambda, \beta \in \Lambda$ and for any $i, j \in I, a_{\lambda}+i$ is adjacent to $a_{\beta}+j$ if and only if $a_{\lambda}+I$ is adjacent to $a_{\beta}+I$ in $T\left(\Gamma(R / I)\right.$ ) (i.e., $a_{\lambda}+a_{\beta} \in S(I)$ ),

(3) for $\lambda \in \Lambda$ and distinct $i, j \in I, a_{\lambda}+i$ is adjacent to $a_{\lambda}+j$ if and only if $2 a_{\lambda} \in S(I)$.

It follows that if $T(\Gamma(R / I))$ is a graph on $N=|R / I|$ vertices, then $T\left(\Gamma_{I}(R)\right)$ is a graph on $N .|I|$ vertices.

Proposition 2.7. Let $R$ be a commutative ring with the proper ideal $I$. Then

(1) $S\left(\Gamma_{I}(R)\right)$ is complete (connected) if and only if $Z(\Gamma(R / I))$ is complete (connected).

(2) If $\bar{S}\left(\Gamma_{I}(R)\right)$ is complete, then $\operatorname{Reg}(\Gamma(R / I))$ is complete.

(3) $\bar{S}\left(\Gamma_{I}(R)\right)$ is connected if and only if $\operatorname{Reg}(\Gamma(R / I))$ is connected.

Proof. (1) Let $S\left(\Gamma_{I}(R)\right)$ be a complete subgraph $T\left(\Gamma_{I}(R)\right)$ and $x+I \neq y+I$ are distinct elements of $Z(\Gamma(R / I))$. So $x$ and $y$ are adjacent in $S\left(\Gamma_{I}(R)\right)$; hence $x+I$ and $y+I$ are adjacent in $Z(\Gamma(R / I))$. Conversely, suppose $x$ and $y$ are distinct elements of $S\left(\Gamma_{I}(R)\right)$. If $x+I=y+I$, then $x-y \in I$. There exists $r \in R-I$ such that $r y \in I$; hence $r x \in I$. It follows that $r(x+y) \in I$, thus $x$ and $y$ are adjacent in $S\left(\Gamma_{I}(R)\right)$. If $x+I \neq y+I$, then $x+I$ and $y+I$ are adjacent in $Z(\Gamma(R / I))$. So $x+y \in S(I)$, as required.

(2) The proof is omitted. The converse is not necessarily true, for example consider $R=\mathbb{Z}_{18}$, and $I=\langle\overline{3}\rangle$ (it is easy to check that $S(I)=I$ ).

(3)The sufficiency implication is clear. Let $\operatorname{Reg}(\Gamma(R / I))$ is connected. Suppose $x$ and $y$ are distinct elements of $\bar{S}\left(\Gamma_{I}(R)\right)$. If $x+I=y+I$, then $x-(-y)-y$ is a path between $x$ and $y$ (if $x=-y$, then $x$ and $y$ are adjacent). If $x+I \neq y+I$, the proof is clear and omitted.

Lemma 2.8. Let $R$ be a commutative ring with the proper ideal $I$. Then $\operatorname{gr}\left(T\left(\Gamma_{I}(R)\right)\right) \leq \operatorname{gr}(T(\Gamma(R / I)))$. If $T(\Gamma(R / I))$ contains a cycle, then so does $T\left(\Gamma_{I}(R)\right)$, and therefore $\operatorname{gr}\left(T\left(\Gamma_{I}(R)\right)\right) \leq \operatorname{gr}(T(\Gamma(R / I))) \leq 4$.

Proof. If $\operatorname{gr}(T(\Gamma(R / I)))=\infty$ we are done. Now suppose $\operatorname{gr}(T(\Gamma(R / I)))=k<$ $\infty$. Let $x_{1}+I-x_{2}+I-\cdots-x_{k}+I-x_{1}+I$ be a cycle in $T(\Gamma(R / I))$ through $k$ 
distinct vertices. Thus $x_{1}-x_{2}-\cdots-x_{k}-x_{1}$ is a cycle in $T\left(\Gamma_{I}(R)\right)$ of length $k$. Hence, $\operatorname{gr}\left(T\left(\Gamma_{I}(R)\right)\right) \leq k$. According to $[2$, Theorem 2.6(3), 3.15(2)], it follows that $\operatorname{gr}(T(\Gamma(R / I))) \leq 4$.

\section{The case when $S(I)$ is an ideal of $R$}

In this section, we state a general structure for $\bar{S}\left(\Gamma_{I}(R)\right)$ the (induced) subgraph $T\left(\Gamma_{I}(R)\right)$ (see Theorem 3.5) and we investigate the relationship between $T\left(\Gamma_{I}(R)\right)$ and $T(\Gamma(R / I))$ with assumption that, $S(I)$ be an ideal of $R$ (i.e., $I$ is a primal ideal of $R$ ). We begin with the following theorem.

Proposition 3.1. Let $R$ be a commutative ring with the proper ideal I such that $S(I)$ is an ideal of $R$. Then $S\left(\Gamma_{I}(R)\right)$ is a complete (induced) subgraph $T\left(\Gamma_{I}(R)\right)$ and is disjoint from $\bar{S}\left(\Gamma_{I}(R)\right)$.

Proof. This is clear according to definition.

Theorem 3.2. Let $R$ be a commutative ring with the proper ideal I such that $S(I)$ is an ideal of $R$.

(1) The (induced) subgraph $S\left(\Gamma_{I}(R)\right)$ with vertices $\sqrt{I}$ is complete and each vertex of this subgraph is adjacent to each vertex of $S\left(\Gamma_{I}(R)\right)$ and is disjoint from $\bar{S}\left(\Gamma_{I}(R)\right)$.

(2) If $\{0\} \neq \sqrt{I} \subset S(I)$, then $\operatorname{gr}\left(S\left(\Gamma_{I}(R)\right)\right)=3$.

Proof. (1) Let $x \in \sqrt{I}$. If $x \in I$, then $x \in S(I)$; otherwise there is an integer $n \geq 2$ such that $x^{n} \in I$ and $x^{n-1} \notin I$. We have $x . x^{n-1} \in I$; hence $x \in S(I)$. So Part (1) follows since $\sqrt{I} \subseteq S(I)$ is an ideal and $\sqrt{I}+S(I) \subseteq S(I)$.

(2) Let $0 \neq x \in \sqrt{I}$ and $y \in S(I) \backslash \sqrt{I}$. Then $0-x-y-0$ is a 3 -cycle in $S\left(\Gamma_{I}(R)\right)$, as required.

Theorem 3.3. Let $R$ be a commutative ring with the proper ideal I such that $S(I)$ is an ideal of $R$.

(1) Assume that $\Gamma$ is an induced subgraph of $\bar{S}\left(\Gamma_{I}(R)\right)$ and let $x$ and $y$ be distinct vertices of $\Gamma$ such that are connected by a path in $\Gamma$. Then there exists a path in $\Gamma$ of length 2 between $x$ and $y$. In particular, if $\bar{S}\left(\Gamma_{I}(R)\right)$ is connected, then $\operatorname{diam}\left(\bar{S}\left(\Gamma_{I}(R)\right)\right) \leq 2$.

(2) Suppose $x$ and $y$ are distinct elements of $\bar{S}\left(\Gamma_{I}(R)\right)$ that are connected by a path. If $x+y \notin S(I)$ (that is, if $x$ and $y$ are not adjacent), then $x-(-x)-y$ and $x-(-y)-y$ are paths of length 2 between $x$ and $y$ in $\bar{S}\left(\Gamma_{I}(R)\right)$.

Proof. (1) Let $x_{1}, x_{2}, x_{3}$, and $x_{4}$ are distinct vertices of $\Gamma$. It suffices to show that if there is a path $x_{1}-x_{2}-x_{3}-x_{4}$ from $x_{1}$ to $x_{4}$, then $x_{1}$ and $x_{4}$ are adjacent. So $x_{1}+x_{2}, x_{2}+x_{3}, x_{3}+x_{4} \in S(I)$ gives $x_{1}+x_{4}=\left(x_{1}+x_{2}\right)-\left(x_{2}+x_{3}\right)+\left(x_{3}+x_{4}\right) \in$ $S(I)$ since $S(I)$ is an ideal of $R$. Thus $x_{1}$ and $x_{4}$ are adjacent. So if $\bar{S}\left(\Gamma_{I}(R)\right)$ is connected, then $\operatorname{diam}\left(\bar{S}\left(\Gamma_{I}(R)\right)\right) \leq 2$.

(2) Since $x, y \in R-S(I)$ and $x+y \notin S(I)$, there exists $z \in R-S(I)$ such that $x-z-y$ is a path of length 2 by part (1) above. Thus $x+z, z+y \in S(I)$, 
and hence $x-y=(x+z)-(z+y) \in S(I)$. Also, since $x+y \notin S(I)$, we must have $x \neq-x$ and $y \neq-x$. Thus $x-(-x)-y$ and $x-(-y)-y$ are paths of length 2 between $x$ and $y$ in $\bar{S}\left(\Gamma_{I}(R)\right)$.

Theorem 3.4. Let $R$ be a commutative ring with the proper ideal $I$ such that $S(I)$ is an ideal of $R$. Then the following statements are equivalent.

(1) $\bar{S}\left(\Gamma_{I}(R)\right)$ is connected.

(2) Either $x+y \in S(I)$ or $x-y \in S(I)$ for all $x, y \in R-S(I)$.

(3) Either $x+y \in S(I)$ or $x+2 y \in S(I)$ (but not both) for all $x, y \in R-S(I)$. In particular, either $2 x \in S(I)$ or $3 x \in S(I)$ for all $x \in R-S(I)$.

Proof. $(1) \Longrightarrow(2)$ Let $x, y \in R-S(I)$ be such that $x+y \notin S(I)$. If $x=y$, then $x-y \in S(I)$. Otherwise, $x-(-y)-y$ is a path from $x$ to $y$ by Theorem $3.3(2)$, and hence $x-y \in S(I)$.

$(2) \Longrightarrow(3)$ Let $x, y \in R-S(I)$, and suppose that $x+y \notin S(I)$. By assumption, since $(x+y)-y=x \notin S(I)$, we have $x+2 y=(x+y)+y \in S(I)$. Let $x+y$ and $x+2 y$ belong to $S(I)$. Then $y \in S(I)$ a contradiction. In particular, if $x \in R-S(I)$, then either $2 x \in S(I)$ or $3 x \in S(I)$.

$(3) \Longrightarrow(1)$ Let $x, y \in R-S(I)$ be distinct elements of $R$ such that $x+y \notin S(I)$. By assumption, since $S(I)$ is an ideal of $R$ and $x+2 y \in S(I)$, we get $2 y \notin S(I)$. Thus $3 y \in S(I)$ by hypothesis. Since $x+y \notin S(I)$ and $3 y \in S(I)$, we conclude that $x \neq 2 y$, and hence $x-2 y-y$ is a path from $x$ to $y$ in $\bar{S}\left(\Gamma_{I}(R)\right)$. Thus $\bar{S}\left(\Gamma_{I}(R)\right)$ is connected.

Theorem 3.5. Let $R$ be a commutative ring with the proper ideal $I$ such that $S(I)$ is an ideal of $R$, and let $|S(I)|=\alpha$ and $|R / S(I)|=\beta$ (we allow $\alpha$ and $\beta$ to be infinite, then we have $\beta-1=(\beta-1) / 2=\beta)$.

(1) If $2 \in S(I)$, then $\bar{S}\left(\Gamma_{I}(R)\right)$ is the union of $\beta-1$ disjoint $K^{\alpha,}$ s.

(2) If $2 \notin S(I)$, then $\bar{S}\left(\Gamma_{I}(R)\right)$ is the union of $(\beta-1) / 2$ disjoint $K^{\alpha, \alpha}$ s.

Proof. (1) Suppose that $2 \in S(I)$, and let $x \in R-S(I)$. Note that each coset $x+S(I)$ is a complete subgraph of $\bar{S}\left(\Gamma_{I}(R)\right)$ since $\left(x+x_{1}\right)+\left(x+x_{2}\right)=$ $2 x+x_{1}+x_{2} \in S(I)$ for all $x_{1}, x_{2} \in S(I)$. We must have that distinct cosets form disjoint subgraphs of $\bar{S}\left(\Gamma_{I}(R)\right)$ since if $x+x_{1}$ and $y+x_{2}$ are adjacent for some $x, y \in R-S(I)$ and $x_{1}, x_{2} \in S(I)$, then $x+y=\left(x+x_{1}\right)+\left(y+x_{2}\right)-\left(x_{1}+x_{2}\right) \in$ $S(I)$, and hence $x-y=(x+y)-2 y \in S(I)$ since $S(I)$ is an ideal $R$ and $2 \in S(I)$. But then $x+S(I)=y+S(I)$. Thus $\bar{S}\left(\Gamma_{I}(R)\right)$ is the union of $\beta-1$ disjoint (induced) subgraphs $x+S(I)$, each of which is a $K^{\alpha}$, where $\alpha=|S(I)|=|x+S(I)|$.

(2) Let $x \in R-S(I)$ and $2 \notin S(I)$. Then no two distinct elements in $x+S(I)$ are adjacent; otherwise if $\left(x+x_{1}\right)+\left(x+x_{2}\right) \in S(I)$ for $x_{1}, x_{2} \in S(I)$ implies that $2 x \in S(I)$, and hence $2 \in S(I)$, a contradiction.

On the other hand, the two cosets $x+S(I)$ and $-x+S(I)$ are disjoint, and each element of $x+S(I)$ is adjacent to each element of $-x+S(I)$. Thus $(x+S(I)) \cup(-x+S(I))$ is a complete bipartite (induced) subgraph of $\bar{S}\left(\Gamma_{I}(R)\right)$; 
furthermore, if $y+x_{1}$ adjacent to $x+x_{2}$ for some $y \in R-S(I)$ and $x_{1}, x_{2} \in S(I)$, then $x+y \in S(I)$, and hence $y+S(I)=-x+S(I)$. Thus $\bar{S}\left(\Gamma_{I}(R)\right)$ is the union of $(\beta-1) / 2$ disjoint (induced) subgraphs $(x+S(I)) \cup(-x+S(I))$, each of which is a $K^{\alpha, \alpha}$, where $\alpha=|S(I)|=|x+S(I)|$.

Remark 3.6. If $S(I)$ is an ideal of $R$, according to Note 2.1, $Z(R / I)=S(I) / I$. Let $|Z(R / I)|=\alpha^{\prime}$ and $|R / I / Z(R / I)|=\beta^{\prime}$. With the above notation, it is easy to check that $\alpha=\alpha^{\prime}|I|$ and $\beta=\beta^{\prime} .2+I \in Z(R / I)$ if and only if $2 \in S(I)$. Let $2 \in S(I)$. By part (1) of the above theorem and [2, Theorem 2.2(1)], $\bar{S}\left(\Gamma_{I}(R)\right)$ is the union of $\beta-1$ disjoint $K^{\alpha,} s$ and $\operatorname{Reg}(\Gamma(R / I))$ is the union of $\beta-1$ disjoint $K^{\alpha /|I|}, s$. Let $2 \notin S(I)$. By part (2) of the above theorem and [2, Theorem 2.2(2)], $\bar{S}\left(\Gamma_{I}(R)\right)$ is the union of $(\beta-1) / 2$ disjoint $K^{\alpha, \alpha}, s$ and $\operatorname{Reg}(\Gamma(R / I))$ is the union of $(\beta-1) / 2$ disjoint $K^{\alpha /|I|, \alpha /|I|} s$. It follows from Remark 2.5, $S\left(\Gamma_{I}(R)\right)$ contains $|I|$ disjoint subgraphs isomorphic to $Z(\Gamma(R / I))$ and $\bar{S}\left(\Gamma_{I}(R)\right)$ contains $|I|$ disjoint subgraphs isomorphic to $\operatorname{Reg}(\Gamma(R / I))$.

Example 3.7. Let $n \geq 2$ be an integer. Then $Z\left(Z_{n}\right)$ is an ideal $Z_{n}$ if and only if $n=p^{k}$ for some prime $p$ and integer $k \geq 1$ (see, [2, Example 2.7]). Let $\langle n\rangle=n \mathbb{Z}$. Since $Z(\mathbb{Z} /\langle n\rangle)=\{a+\langle n\rangle: a \in S(\langle n\rangle)\}$; hence $S(\langle n\rangle)$ is an ideal $\mathbb{Z}$ if and only if $n=p^{k}$ for some prime $p$ and integer $k \geq 1$. Let $n=p^{k}$ for some prime $p$ and integer $k \geq 1$. It is easy to check that $S\left(\left\langle p^{k}\right\rangle\right)=\langle p\rangle$, that is $\left\langle p^{k}\right\rangle$ is a $p \mathbb{Z}$-primal ideal $\mathbb{Z}$. If $p=2$, then $\bar{S}\left(\Gamma_{\left\langle p^{k}\right\rangle}(\mathbb{Z})\right)$ is the complete subgraph $K^{\alpha,} s$ such that $|\langle p\rangle|=\alpha$. If $p>2$, then $\bar{S}\left(\Gamma_{\left\langle p^{k}\right\rangle}(\mathbb{Z})\right)$ is the union of $p-1 / 2$ disjoint $K^{\alpha, \alpha,} s$.

Note 3.8. Note that if $S(I)=\{0\}$, then $R$ is an integral domain, and $2 \in S(I)$ if and only if char $R=2$.

Theorem 3.9. Let $R$ be a commutative ring with the proper ideal I such that $S(I)$ is an ideal of $R$. Then

(1) $\bar{S}\left(\Gamma_{I}(R)\right)$ is complete if and only if $R / S(I) \cong \mathbb{Z}_{2}$ or $R \cong \mathbb{Z}_{3}$.

(2) $\bar{S}\left(\Gamma_{I}(R)\right)$ is connected if and only if $R / S(I) \cong \mathbb{Z}_{2}$ or $R / S(I) \cong \mathbb{Z}_{3}$.

(3) $\bar{S}\left(\Gamma_{I}(R)\right)$ (and hence $T\left(\Gamma_{I}(R)\right)$ and $S\left(\Gamma_{I}(R)\right)$ ) is totally disconnected if and only if $I=\{0\}$ and $R$ is an integral domain, with char $R=2$.

Proof. Let $|S(I)|=\alpha$ and $|R / S(I)|=\beta$.

(1) $\bar{S}\left(\Gamma_{I}(R)\right)$ is complete if and only if $\bar{S}\left(\Gamma_{I}(R)\right)$ is a single $K^{\alpha}$ or $K^{1,1}$ by Theorem 3.5.

Let $\bar{S}\left(\Gamma_{I}(R)\right)$ be a complete subgraph of $T\left(\Gamma_{I}(R)\right)$. If $2 \in S(I)$, then $\beta-1=$ 1. Thus $R / S(I) \cong \mathbb{Z}_{2}$. If $2 \notin S(I)$, then $\alpha=1$ and $(\beta-1) / 2=1$. Thus $S(I)=\{0\}=I$ and $\beta=3$; hence $R \cong \mathbb{Z}_{3}$.

Conversely, if $R / S(I) \cong \mathbb{Z}_{2}$, then we show that $2 \in S(I) . R / S(I)=$ $\{S(I), x+S(I)\}$ where $x \notin S(I)$. Thus $x+S(I)=-x+S(I)$ gives $2 x \in S(I)$; hence $2 \in S(I)$. So, $\bar{S}\left(\Gamma_{I}(R)\right)$ is a single $K^{\alpha}$. Next, suppose that $R \cong \mathbb{Z}_{3}$, then $I=\{0\}$ is only proper ideal of $R$, since $T\left(\Gamma_{0}(R)\right)=T(\Gamma(R))$, as required. 
(2) By Theorem 3.5, $\bar{S}\left(\Gamma_{I}(R)\right)$ is a connected subgraph $T\left(\Gamma_{I}(R)\right)$ if and only if $\bar{S}\left(\Gamma_{I}(R)\right)$ is a single $K^{\alpha}$ or $K^{\alpha, \alpha}$. Let $\bar{S}\left(\Gamma_{I}(R)\right)$ be a connected subgraph of $T\left(\Gamma_{I}(R)\right)$. If $2 \in S(I)$, then $\beta-1=1$. Thus $R / S(I) \cong \mathbb{Z}_{2}$. If $2 \notin S(I)$, then $\beta-1 / 2=1$ gives $\beta=3$; hence $R / S(I) \cong \mathbb{Z}_{3}$.

Conversely, by part (1), it suffices to show that $\bar{S}\left(\Gamma_{I}(R)\right)$ is connected when $R / S(I) \cong \mathbb{Z}_{3}$. We claim that $2 \notin S(I)$. Suppose not. Then $R / S(I)=$ $\{S(I), x+S(I), y+S(I)\}$ where $x, y \notin S(I)$. Since $R / S(I)$ is a cyclic group with order of 3 , we have $(x+S(I))+(x+S(I))=y+S(I)$; hence $y \in S(I)$ $(2 x \in S(I))$, a contradiction. Thus $2 \notin S(I)$ and by Theorem $3.5(2), \bar{S}\left(\Gamma_{I}(R)\right)$ is a single $K^{\alpha, \alpha}$ and the proof is complete.

(3) $\bar{S}\left(\Gamma_{I}(R)\right)$ is totally disconnected if and only if it is a disjoint union of $K^{1,} s$. Hence by Theorem 3.5, $2 \in S(I)$ and $|S(I)|=1$. So $R$ must be an integral domain with char $R=2$.

Remark 3.10. Let $S(I)$ be an ideal. Then $R / I / Z(R / I)=R / I / S(I) / I \cong$ $R / S(I)$; hence $R / I / Z(R / I) \cong \mathbb{Z}_{n}$ if and only if $R / S(I) \cong \mathbb{Z}_{n}$ such that $n \geq 2$ is an integer. So the above theorem in conjunction with [2, Theorem 2.4] is the other proof of Proposition 2.7.

At the end of this section, we give further explicit descriptions of the diameter and girth of $\bar{S}\left(\Gamma_{I}(R)\right)$.

Proposition 3.11. Let $R$ be a commutative ring with proper ideal I such that $S(I)$ is an ideal of $R$. Then

(1) $\operatorname{diam}\left(\bar{S}\left(\Gamma_{I}(R)\right)\right)=0,1,2$, or $\infty$. In particular, $\operatorname{diam}\left(\bar{S}\left(\Gamma_{I}(R)\right)\right) \leq 2$ if $\bar{S}\left(\Gamma_{I}(R)\right)$ is connected.

(2) $\operatorname{gr}\left(\bar{S}\left(\Gamma_{I}(R)\right)\right)=3,4$ or $\infty$. In particular, $\operatorname{gr}\left(\bar{S}\left(\Gamma_{I}(R)\right)\right) \leq 4$ if $\left.\bar{S}\left(\Gamma_{I}(R)\right)\right)$ contains a cycle.

Proof. (1) Suppose that $\bar{S}\left(\Gamma_{I}(R)\right)$ is connected. Then $\bar{S}\left(\Gamma_{I}(R)\right)$ is a singleton, a complete graph, or a complete bipartite graph by Theorem 3.5. Thus $\operatorname{diam}\left(\bar{S}\left(\Gamma_{I}(R)\right)\right) \leq 2$.

(2) Let $\bar{S}\left(\Gamma_{I}(R)\right)$ contains a cycle. Since $\left.\bar{S}\left(\Gamma_{I}(R)\right)\right)$ is a disjoint union of either complete or complete bipartite graphs by Theorem 3.5, it must contain either a 3 -cycle or a 4-cycle. Thus $\operatorname{gr}\left(\bar{S}\left(\Gamma_{I}(R)\right)\right) \leq 4$.

Theorem 3.12. Let $R$ be a commutative ring with the proper ideal I such that $S(I)$ is an ideal of $R$.

(1) $\operatorname{diam}\left(\bar{S}\left(\Gamma_{I}(R)\right)\right)=0$ if and only if $R \cong \mathbb{Z}_{2}$.

(2) $\operatorname{diam}\left(\bar{S}\left(\Gamma_{I}(R)\right)\right)=1$ if and only if either $R / S(I) \cong \mathbb{Z}_{2}$ and $|S(I)| \geq 2$ or $R \cong \mathbb{Z}_{3}$.

(3) $\operatorname{diam}\left(\bar{S}\left(\Gamma_{I}(R)\right)\right)=2$ if and only if $R / S(I) \cong \mathbb{Z}_{3}$ and $|S(I)| \geq 2$.

(4) Otherwise, $\operatorname{diam}\left(\bar{S}\left(\Gamma_{I}(R)\right)\right)=\infty$.

Proof. These results all follow from Theorem 3.5, Theorem 3.9 and Proposition 3.11 . 
Corollary 3.13. Let $S(I)$ be an ideal of $R$ and $I \neq 0$. Then we have the following results:

(1) If $\operatorname{diam}(\operatorname{Reg}(\Gamma(R / I)))=0$, then $\operatorname{diam}\left(\bar{S}\left(\Gamma_{I}(R)\right)\right)=1$ and $I=S(I)$.

(2) Let $\operatorname{diam}(\operatorname{Reg}(\Gamma(R / I)))=1$. Then $\operatorname{diam}\left(\bar{S}\left(\Gamma_{I}(R)\right)\right)=1$ if $I \subsetneq S(I)$ and $\operatorname{diam}\left(\bar{S}\left(\Gamma_{I}(R)\right)\right)=2$ if $I=S(I)$.

(3) If $\operatorname{diam}(\operatorname{Reg}(\Gamma(R / I)))=2$, then $\operatorname{diam}\left(\bar{S}\left(\Gamma_{I}(R)\right)\right)=2$.

(4) $\operatorname{diam}\left(\bar{S}\left(\Gamma_{I}(R)\right)\right)=\infty$ if and only if $\operatorname{diam}(\operatorname{Reg}(\Gamma(R / I)))=\infty$.

Proof. These results all follow directly from Remark 3.10, Theorem 3.12 and [2, Theorem 2.6(1)]. Note that for (4), $\operatorname{diam}\left(\bar{S}\left(\Gamma_{I}(R)\right)\right)=\infty$ if and only if $2 \in S(I)$ and $|R / S(I)|=\beta \geq 3$, or $2 \notin S(I)$ and $|R / S(I)|=\beta \geq 5$. So, by Note 2.1 and $[2$, Theorem 2.2], the proof is complete.

Corollary 3.14. Let $S(I)$ be an ideal of $R$ and $I \subsetneq S(I)$. If $\operatorname{diam}\left(\bar{S}\left(\Gamma_{I}(R)\right)\right)=$ $k$ such that $0 \leq k \leq 2$ is an integer, then $\operatorname{diam}(\operatorname{Reg}(\Gamma(R / I)))=k$.

Proof. The result follows by Remark 3.10, Theorem 3.12 and [2, Theorem 2.6(1)].

Theorem 3.15. Suppose that $S(I)$ is an ideal of $R$. Then

(1) (a) $\operatorname{gr}\left(\bar{S}\left(\Gamma_{I}(R)\right)\right)=3$ if and only if $2 \in S(I)$ and $|S(I)| \geq 3$.

(b) $\operatorname{gr}\left(\bar{S}\left(\Gamma_{I}(R)\right)\right)=4$ if and only if $2 \notin S(I)$ and $|S(I)| \geq 2$.

(c) Otherwise, $\operatorname{gr}\left(\bar{S}\left(\Gamma_{I}(R)\right)\right)=\infty$.

(2) (a) $\operatorname{gr}\left(T\left(\Gamma_{I}(R)\right)\right)=3$ if and only if $|S(I)| \geq 3$.

(b) $\operatorname{gr}\left(T\left(\Gamma_{I}(R)\right)\right)=4$ if and only if $2 \notin S(I)$ and $|S(I)|=2$.

(c) Otherwise, $\operatorname{gr}\left(T\left(\Gamma_{I}(R)\right)\right)=\infty$.

Proof. According to Theorem 3.1, Theorem 3.5, these results follow.

Corollary 3.16. Let $S(I)$ be an ideal of $R$. Then

(1) (a) If $\operatorname{gr}(\operatorname{Reg}(\Gamma(R / I)))=k$ such that $3 \leq k \leq 4$ is an integer, then $\operatorname{gr}\left(\bar{S}\left(\Gamma_{I}(R)\right)\right)=k$.

(b) If $\{0\} \neq I \subsetneq S(I)$ and $\operatorname{gr}(\operatorname{Reg}(\Gamma(R / I)))=\infty$, then $\operatorname{gr}\left(\bar{S}\left(\Gamma_{I}(R)\right)\right)=$ 3 .

(2) (a) If $\operatorname{gr}\left(\bar{S}\left(\Gamma_{I}(R)\right)\right)=3$, then if $|Z(R / I)| \leq 2, \operatorname{gr}\left(\bar{S}\left(\Gamma_{I}(R)\right)\right)=\infty$. If $|Z(R / I)|>2$, then $\operatorname{gr}\left(\bar{S}\left(\Gamma_{I}(R)\right)\right)=3$.

(b) If $\operatorname{gr}\left(\bar{S}\left(\Gamma_{I}(R)\right)\right)=4$, then $\operatorname{gr}(\operatorname{Reg}(\Gamma(R / I)))=4$, if $I \subsetneq S(I)$; otherwise $\operatorname{gr}(\operatorname{Reg}(\Gamma(R / I)))=\infty$.

(c) If $\operatorname{gr}\left(\bar{S}\left(\Gamma_{I}(R)\right)\right)=\infty$, then $\operatorname{gr}(\operatorname{Reg}(\Gamma(R / I)))=\infty$.

Proof. These results all follow directly from Note 2.1, Remark 3.10, and Theorem 3.15 and [2, Theorem 2.6(2)].

\section{The case when $S(I)$ is not an ideal $R$}

Given a proper ideal $I$ of $R$, in this section we study the remaining case when $S(I)$ is not an ideal of $R$ (i.e., $I$ is not primal ideal of $R$ ). Since $S(I)$ is always 
closed under product by elements of $R$; hence there are distinct $x, y \in S(I)^{*}$ such that $x+y \in R-S(I)$, so $|S(I)| \geq 3$; in this case, $S\left(\Gamma_{I}(R)\right)$ and $\bar{S}\left(\Gamma_{I}(R)\right)$ are never disjoint subgraphs. Also, we determine when $T\left(\Gamma_{I}(R)\right)$ is connected and compute $\operatorname{diam}\left(T\left(\Gamma_{I}(R)\right)\right)$.

Theorem 4.1. Suppose that $S(I)$ is not an ideal of $R$.

(1) $S\left(\Gamma_{I}(R)\right)$ is connected with $\operatorname{diam}\left(S\left(\Gamma_{I}(R)\right)\right)=2$.

(2) Some vertex of $S\left(\Gamma_{I}(R)\right)$ is adjacent to a vertex of $\bar{S}\left(\Gamma_{I}(S)\right)$.

In particular, the subgraphs $S\left(\Gamma_{I}(R)\right)$ and $\bar{S}\left(\Gamma_{I}(S)\right)$ are not disjoint.

(3) If $\bar{S}\left(\Gamma_{I}(S)\right)$ is connected, then $T\left(\Gamma_{I}(S)\right)$ is connected.

Proof. (1) Let $x \in S(I)^{*}$. Then $x$ is adjacent to 0 . Thus $x-0-y$ is a path in $S\left(\Gamma_{I}(R)\right)$ of length two between any two distinct $x, y \in S(I)^{*}$. Moreover, there exist nonadjacent $x, y \in S(I)^{*}$ since $S(I)$ is not an ideal of $R$; thus $\operatorname{diam}\left(S\left(\Gamma_{I}(R)\right)\right)=2$.

(2) By assumption, there exist distinct $x, y \in S(I)^{*}$ such that $x+y \notin S(I)^{*}$; so $x+y \in R-S(I)$. Then $-x \in S(I)$ and $x+y \in R-S(I)$ are adjacent vertices in $T\left(\Gamma_{I}(R)\right)$ since $-x+(x+y)=y \in S(I)$. The "in particular" statement is clear.

(3) By part (1) above, it suffices to show that there is a path from $x$ to $y$ in $T\left(\Gamma_{I}(R)\right)$ for any $x \in S(I)$ and $y \in R-S(I)$. By part (2) above, there exist adjacent vertices $u$ and $v$ in $S\left(\Gamma_{I}(R)\right)$ and $\bar{S}\left(\Gamma_{I}(R)\right)$, respectively. Since $S\left(\Gamma_{I}(R)\right)$ is connected, there is a path from $x$ to $u$ in $S\left(\Gamma_{I}(R)\right)$; and since $\bar{S}\left(\Gamma_{I}(R)\right)$ is connected, there is a path from $v$ to $y$ in $\bar{S}\left(\Gamma_{I}(R)\right)$. Then there is a path from $x$ to $y$ in $T\left(\Gamma_{I}(R)\right)$ since $u$ and $v$ are adjacent in $T\left(\Gamma_{I}(R)\right)$. It follows that, $T\left(\Gamma_{I}(R)\right)$ is connected.

The Jacobson radical $\operatorname{Jac}(R)$ of $R$ is defined to be the intersection of all the maximal ideal of $R$, [4, Proposition 1.9]. Consider the following lemma.

Lemma 4.2. Suppose that $S(I)$ is not an ideal of $R$. Then $T\left(\Gamma_{I}(R)\right)$ is connected if and only if $R=\left\langle a_{1}, \ldots, a_{k}\right\rangle$ for some $a_{1}, \ldots, a_{k} \in S(I)$. In particular, if $R / I$ is a finite ring and $I \subseteq \operatorname{Jac}(R)$, then $T\left(\Gamma_{I}(R)\right)$ is connected.

Proof. Suppose $T\left(\Gamma_{I}(R)\right)$ is connected. Hence there is a path $0-x_{1}-\cdots-x_{n}-1$ from 0 to 1 in $T\left(\Gamma_{I}(R)\right)$. Now $x_{1}, x_{1}+x_{2}, \ldots, x_{n}+1 \in S(I)$. Hence $1 \in$ $\left\langle x_{1}, x_{1}+x_{2}, \ldots, x_{n-1}+x_{n}, x_{n}+1\right\rangle \subseteq\langle S(I)\rangle$; thus $R=\langle S(I)\rangle$. Conversely, suppose that $R=\langle S(I)\rangle$. We show that for each $0 \neq x \in R$, there exists a path in $T\left(\Gamma_{I}(R)\right)$ from 0 to $x$. By assumption, there are elements $z_{1}, \ldots, z_{n} \in S(I)$ such that $x=z_{1}+\cdots+z_{n}$. Set $w_{0}=0$ and $w_{k}=(-1)^{n+k}\left(z_{1}+\cdots+z_{k}\right)$ for each integer $k$ with $1 \leq k \leq n$. Then $w_{k}+w_{k+1}=(-1)^{n+k+1} z_{k+1} \in S(I)$ for each integer $k$ with $0 \leq k \leq n-1$; and thus $0-w_{1}-w_{2}-\cdots-w_{n-1}-w_{n}=x$ is a path from 0 to $x$ in $T\left(\Gamma_{I}(R)\right)$ of length at most $n$. Now let $0 \neq u, v \in R$. Then by the preceding argument, there are paths from $u$ to 0 and 0 to $v$ in $T\left(\Gamma_{I}(R)\right)$; hence there is a path from $u$ to $v$ in $T\left(\Gamma_{I}(R)\right)$. Thus, $T\left(\Gamma_{I}(R)\right)$ is connected. 
In the light of Lemma 4.2, we have the following results.

Theorem 4.3. Suppose that $S(I)$ is not an ideal of $R$ and $R=\langle S(I)\rangle$. Let $n \geq 2$ be the least integer such that $R=\left\langle x_{1}, \ldots, x_{n}\right\rangle$ for some $x_{1}, \ldots, x_{n} \in S(I)$ (that is, $T\left(\Gamma_{I}(R)\right)$ is connected). Then diam $\left(T\left(\Gamma_{I}(R)\right)\right)=n$. In particular, if $R / I$ is a finite ring and $I \subseteq \operatorname{Jac}(R)$, then $\operatorname{diam}\left(T\left(\Gamma_{I}(R)\right)\right)=2$.

Proof. First, we investigate any path from 0 to 1 in $T\left(\Gamma_{I}(R)\right)$ has length $\geq n$. Suppose that $0-x_{1}-x_{2}-\cdots-x_{m-1}-1$ is a path from 0 to 1 in $T\left(\Gamma_{I}(R)\right)$ of length $m$. Thus $x_{1}, x_{1}+x_{2}, \ldots, x_{m-2}+x_{m-1}, x_{m-1}+1 \in S(I)$, and hence $1 \in\left(x_{1}, x_{1}+x_{2}, \ldots, x_{m-2}+x_{m-1}, x_{m-1}+1\right) \subseteq(S(I))$. Thus $m \geq n$.

Now, let $x$ and $y$ be distinct elements in $R$. We show that there is a path from $x$ to $y$ in $T\left(\Gamma_{I}(R)\right)$ with length $\leq n$. Let $1=b_{1}+\cdots+b_{n}$ for some $b_{1}, \ldots, b_{n} \in S(I)$, and let $z=y+(-1)^{n+1} x$. Define $w_{0}=x$ and $w_{k}=$ $(-1)^{n+k} z\left(b_{1}+\cdots+b_{k}\right)+(-1)^{k} x$ for each integer $k$ with $1 \leq k \leq n$. Then $w_{k}+w_{k+1}=(-1)^{n+k+1} z b_{k+1} \in S(I)$ for each integer $k$ with $0 \leq k \leq n-1$ and $w_{n}=z+(-1)^{n} x=y$. Thus $x-w_{1}-\cdots-w_{n-1}-y$ is a path from $x$ to $y$ in $T\left(\Gamma_{I}(R)\right)$ with length at most $n$. Specially, we conclude that a shortest path between 0 and 1 in $T\left(\Gamma_{I}(R)\right)$ has length $n$; hence $\operatorname{diam}\left(T\left(\Gamma_{I}(R)\right)\right)=n$. For the "in particular" statement, note that $Z(R / I)$ is not an ideal of $R$. So, $x+y+I \in \operatorname{Reg}(R / I)$ for some $x, y \in S(I)$. Since every regular element of a finite commutative ring is a unit and $I \subseteq \operatorname{Jac}(R)$; hence $x+y$ is a unit. Now, we have $R=\langle x, y\rangle$, and thus $\operatorname{diam}\left(T\left(\Gamma_{I}(R)\right)\right)=2$.

Clearly, if $R=\left\langle a_{1}, \ldots, a_{k}\right\rangle$ for some $a_{1}, \ldots, a_{k} \in S(I)$, then $R / I=\left\langle a_{1}+\right.$ $\left.I, \ldots, a_{k}+I\right\rangle$; hence $\operatorname{diam}(T(\Gamma(R / I))) \leq \operatorname{diam}\left(T\left(\Gamma_{I}(R)\right)\right)$ (see [2, Theorem $3.4]$ ). Note that since, $k \geq 2$ be the least integer such that $R=\left\langle a_{1}, \ldots, a_{k}\right\rangle$; hence $\operatorname{diam}(T(\Gamma(R / I))) \geq \operatorname{diam}\left(T\left(\Gamma_{I}(R)\right)\right)-1$.

Example 4.4. Let $n \geq 2$ be an integer, and let $n \neq p^{k}$ for every prime $p$ and integer $k \geq 1$. Then $S(\langle n\rangle)$ is not an ideal of $\mathbb{Z}$ (see, Example 3.7). It is easy to check that there are distinct primes $p$ and $q$, and integers $r, s \notin\langle n\rangle$ such that $p r \in\langle n\rangle$ and $q s \in\langle n\rangle$. So $\mathbb{Z}=\langle p, q\rangle$; that $p, q \in S(\langle n\rangle)$. By Theorem 4.3, $\operatorname{diam}\left(T\left(\Gamma_{\langle n\rangle}(\mathbb{Z})\right)\right)=2$.

Theorem 4.5. Suppose that $S(I)$ is not an ideal of $R$. If $T\left(\Gamma_{I}(R)\right)$ is connected, then

(1) $\operatorname{diam}\left(T\left(\Gamma_{I}(R)\right)\right)=d(0,1)$.

(2) If $\operatorname{diam}\left(T\left(\Gamma_{I}(R)\right)\right)=n$, then $\operatorname{diam}\left(\bar{S}\left(\Gamma_{I}(R)\right)\right) \geq n-2$.

Proof. (1) This follows from the proof of Theorem 4.3.

(2) By part $(1)$ above, $\operatorname{diam}\left(T\left(\Gamma_{I}(R)\right)\right)=d(0,1)=n$. Let $0-x_{1}-\cdots-$ $x_{n-1}-1$ be a shortest path from 0 to 1 in $T\left(\Gamma_{I}(R)\right)$. Clearly, $x_{1} \in S(I)$. If $x_{i} \in S(I)$ for some integer $i$ with $2 \leq i \leq n-1$, then we can construct the path $0-x_{i}-\cdots-x_{n-1}-1$ from 0 to 1 in $T\left(\Gamma_{I}(R)\right)$ which has length less than $n$, which is a contradiction. Thus $x_{i} \in R-S(I)$ for each integer $i$ with 
$2 \leq i \leq n-1$. Therefore, $x_{2}-x_{3}-\cdots-x_{n-1}-1$ is a shortest path from $x_{2}$ to 1 in $\bar{S}\left(\Gamma_{I}(R)\right)$, and it has length $n-2$. Thus $\operatorname{diam}\left(\bar{S}\left(\Gamma_{I}(R)\right)\right) \geq n-2$.

Corollary 4.6. Let $\left\{R_{\alpha}\right\}_{\alpha \in \Lambda}$ be a family of commutative rings with $|\Lambda| \geq 2$, and let $R=\prod_{\alpha \in \Lambda} R_{\alpha}$. Suppose $I=\prod_{\alpha \in \Lambda} I_{\alpha} ;$ such that for every $\alpha \in \Lambda, I_{\alpha}$ is a proper ideal of $R_{\alpha}$. Then $T\left(\Gamma_{I}(R)\right)$ is connected with $\operatorname{diam}\left(T\left(\Gamma_{I}(R)\right)\right)=2$.

Proof. It is easy to check that $e=(1,0,0, \ldots)$ and $1_{R}-e \in S(I)$. It follows that $R=\left\langle e, 1_{R}-e\right\rangle$; so by Theorem 4.3 , the claim is true.

Remark 4.7. Let $R$ and $U$ be commutative rings, $I$ and $J$ be proper ideals of $R$ and $U$, respectively. It is clear to check that $R \times U-S(I \times J)=(R-S(I)) \times(U-$ $S(J))$. So for distinct $(x, y),(z, w) \in R \times U-S(I \times J),(x, y)-(-x,-w)-(z, w)$ is a path of length at most two in $\bar{S}\left(\Gamma_{I \times J}(R \times U)\right)$. Thus $\bar{S}\left(\Gamma_{I \times J}(R \times U)\right)$ is connected with $\operatorname{diam}\left(\bar{S}\left(\Gamma_{I \times J}(R \times U)\right)\right) \leq 2$. By Theorem $4.1(2)$, it follows that $T\left(\Gamma_{I \times J}(R \times U)\right)$ is connected (see Corollary 4.6).

Theorem 4.8. Let $S(I)$ does not an ideal of $R$. Then $T\left(\Gamma_{S^{-1} I}\left(S^{-1} R\right)\right)$; where $S=R-S(I)$, is connected with $\operatorname{diam}\left(T\left(\Gamma_{S^{-1} I}\left(S^{-1} R\right)\right)\right)=2$. In particular, if $R / I$ is a finite ring and $I \subseteq J a c(R)$, then $\operatorname{diam}\left(T\left(\Gamma_{S^{-1} I}\left(S^{-1} R\right)\right)\right)=2$.

Proof. Since $S(I)$ is not an ideal of $R$, there are $x_{1}, x_{2} \in S(I)$ such that $s=$ $x_{1}+x_{2} \in R-S(I)$. Thus $x_{1} / s+x_{2} / s=1$ in $S^{-1} R$. It is easy to check that $S\left(S^{-1} I\right)$ is not an ideal of $S^{-1} R$ and $x_{1} / s, x_{2} / s \in S\left(S^{-1} I\right)$. Thus $S^{-1} R=$ $\left\langle x_{1} / s, x_{2} / s\right\rangle$. The "in particular" statement is clear since every $s \in S$ is unite $\left(s+I \in \operatorname{Reg}(R / I)\right.$; hence $s+I$ is unite). It follows that $S^{-1} R=R$.

Theorem 4.9. Let $I \unlhd R$, and $P_{1}$ and $P_{2}$ be prime ideals of $R$, containing I. Suppose $x y \in I$ for some $x \in P_{1} \backslash P_{2}$ and $y \in P_{2} \backslash P_{1}$. Then $\operatorname{diam}\left(T\left(\Gamma_{S^{-1} I}\left(R_{S}\right)\right)\right)=2$ where $S=R \backslash P_{1} \cup P_{2}$.

Proof. For all $s \in S$, we have $s x$ and $s y \notin I$; since $s, x \notin P_{2}$ and $s, y \notin P_{1}$. Thus $x / s$ and $y / s$ are nonzero elements of $S\left(S^{-1} I\right)\left((x / s)(y / 1) \in S^{-1} I\right.$ and $y / 1 \notin$ $\left.S^{-1} I\right)$. Let $s=x+y \in S$, hence $S^{-1} R=\langle x / s, y / s\rangle$. Thus $T\left(\Gamma_{S^{-1} I}\left(S^{-1} R\right)\right)$ is connected with $\operatorname{diam}\left(T\left(\Gamma_{S^{-1} I}\left(S^{-1} R\right)\right)\right)=2$ by Theorem 4.3.

The following theorem give $\operatorname{gr}\left(S\left(\Gamma_{I}(R)\right)\right), \operatorname{gr}\left(\bar{S}\left(\Gamma_{I}(R)\right)\right)$, and $\operatorname{gr}\left(T\left(\Gamma_{I}(R)\right)\right)$ when $S(I)$ is not an ideal of $R$.

Theorem 4.10. Let $R$ be a commutative ring with the proper ideal I such that $S(I)$ is not an ideal of $R$. Then

(1) If $I \neq\{0\}, \operatorname{gr}\left(S\left(\Gamma_{I}(R)\right)\right)=3$. Otherwise $\operatorname{gr}\left(S\left(\Gamma_{I}(R)\right)\right)=3$ or $\infty$. Moreover, if $\operatorname{gr}\left(S\left(\Gamma_{I}(R)\right)\right)=\infty$, then $R \cong \mathbb{Z}_{2} \times \mathbb{Z}_{2} ;$ so, $S\left(\Gamma_{I}(R)\right.$ is a $K^{1,2}$ star graph with center 0 .

(2) $\operatorname{gr}\left(T\left(\Gamma_{I}(R)\right)\right)=3$ if and only if $\operatorname{gr}\left(S\left(\Gamma_{I}(R)\right)\right)=3$.

(3) The (induced) subgraph of $S\left(\Gamma_{I}(R)\right)$ with vertices $\sqrt{I}$ is complete; hence $\operatorname{gr}\left(S\left(\Gamma_{I}(R)\right)\right)=3$ when $|\sqrt{I}| \geq 3$.

(4) If $\operatorname{gr}\left(T\left(\Gamma_{I}(R)\right)\right)=4$, then $\operatorname{gr}\left(S\left(\Gamma_{I}(R)\right)\right)=\infty$. 
(5) If $2 \in I$, then $\operatorname{gr}\left(\bar{S}\left(\Gamma_{I}(R)\right)\right)=3$ or $\infty$.

(6) If $2 \notin I$, then $\operatorname{gr}\left(\bar{S}\left(\Gamma_{I}(R)\right)\right)=3,4$ or $\infty$.

Proof. (1) Let $0 \neq x \in I$ and $y \in S(I) \backslash I$. Since $I+S(I) \subseteq S(I), 0-x-y-0$ is a 3 -cycle in $S\left(\Gamma_{I}(R)\right)$. If $I=\{0\}$, it follows from [2, Theorem 3.4(1)]. Note that if $R \cong \mathbb{Z}_{2} \times \mathbb{Z}_{2}$, then $I=\{0\}$ is the only proper ideal of $R$, that $S(I)$ is not an ideal of $R$.

(2) It suffices to show that $\operatorname{gr}\left(S\left(\Gamma_{I}(R)\right)\right)=3$ when $\operatorname{gr}\left(T\left(\Gamma_{I}(R)\right)\right)=3$. If $2 x \neq 0$ for some $x \in S(I)^{*}$, then $0-x-(-x)-0$ is a 3 -cycle in $S(I)$. Thus we may assume that $2 x=0$ for all $x \in S(I)$. Since $S(I)$ is not an ideal; so there are $x \in S(I)$ such that $x \notin I .2 x=0 \in I$; hence $2 \in S(I)$. Let $a-b-c-a$ be a 3 -cycle in $S\left(\Gamma_{I}(R)\right)$. So $a+b, b+c, c+a \in S(I)$. If $2 a=0$, then $0-a+b-a+c-0$ is a 3 -cycle in $S\left(\Gamma_{I}(R)\right)$. So without loss of generality we can assume that $2 a, 2 b$ and $2 c$ are non-zero. If $2 a \neq 2 b$, then $0-2 a-2 b-0$ is a 3 -cycle in $S\left(\Gamma_{I}(R)\right)$. Without loss of generality we can assume that $2 a=2 b=2 c$. So, $2(a-b)=2(b-c)=0 \in I$. If $2 \notin I$, then $a-b$ and $b-c \in S(I)$; hence $0-(a-b)-(b-c)-0$ is a 3 -cycle in $S\left(\Gamma_{I}(R)\right)$ (if $a-b=b-c$, then $a+c=2 b=2 a$, a contradiction). Let $2 \in I$. Since $b+c \in S(I)$; hence $(b+c) r \in I$ such that $r \notin I$; thus $(2 a+b+c) r \in I$. Now $0-a+b-a+c-0$ is a 3 -cycle in $S\left(\Gamma_{I}(R)\right.$ ) (if $a+b=0$, then we have a 3 -cycle $0-a+c-b+c-0)$. Thus in all cases we get a 3 -cycle in $S\left(\Gamma_{I}(R)\right)$.

(3) It follows from $\sqrt{I} \subseteq S(I)$ is an ideal.

(4) It is clear by parts 1,2 .

(5) Let $2 \in I$ and $\bar{S}\left(\Gamma_{I}(R)\right)$ contains a cycle $C$. Hence there is a path $x-y-z$ in $\bar{S}\left(\Gamma_{I}(R)\right)$. Without loss of generality we may assume that $x \neq 1$, $y \neq 1$. Clearly, $x+y, y+z \in S(I)$. Suppose that $R$ contains a $a \in \sqrt{I} \backslash I$. If $a=a x=a y$, then $x+1, y+1 \in S(I)$, and thus $1-x-y-1$ is a 3 -cycle in $\bar{S}\left(\Gamma_{I}(R)\right)$. If either $a x \neq a$ or $a y \neq a$, then either $1-(a+1)-(a x+1)-1$ or $1-(a+1)-(a y+1)-1$ is a 3 -cycle in $\bar{S}\left(\Gamma_{I}(R)\right)(a+I \in J a c(R / I))$. Let $\sqrt{I}=I$. If $I=\{0\}$ (hence $2=0$ ), then $x^{2} \neq y^{2}$; since $x^{2}+y^{2}=(x+y)^{2} \neq 0$. Hence $x^{2}-x y-y^{2}-x^{2}$ is a 3 -cycle in $\bar{S}\left(\Gamma_{I}(R)\right)=\operatorname{Reg}(R)$. Finally, let $I \neq\{0\}$. Suppose $0 \neq b \in I$. If $x+z \in S(I)$, then $x-y-z-x$ is a 3 -cycle in $\bar{S}\left(\Gamma_{I}(R)\right)$. Let $x+z \notin S(I)$. It follows that $y-x$ or $z-y \notin I(2 x \in I)$. Without loss of generality we can assume that $y-x \notin I$; hence $b+x-x-y-b+x$ is a 3 -cycle in $\bar{S}\left(\Gamma_{I}(R)\right)$. So, as required.

(6) Suppose that $\bar{S}\left(\Gamma_{I}(R)\right)$ contains a cycle. So there is a path $x-y-z$ in $\bar{S}\left(\Gamma_{I}(R)\right)$. We may assume that $x+z \notin S(I)$. It is clear that either $x+y \neq 0$ or $y+z \neq 0$ (otherwise $x=z$, a contradiction). Without loss of generality we can assume that $x+y \neq 0$. Then $x-y-(-y)-(-x)-x$ is a 4 -cycle (if $x=-x$ gives $2 x=0 \in I$, then $x \in S(I)$, a contradiction). So, the proof is complete. 


\section{References}

[1] S. Akbari, D. Kiani, F. Mohammadi, and S. Moradi, The total graph and regular graph of a commutative ring, J. Pure Appl. Algebra 213 (2009), no. 12, 2224-2228.

[2] D. F. Anderson and A. Badawi, The total graph of a commutative ring, J. Algebra $\mathbf{3 2 0}$ (2008), no. 7, 2706-2719.

[3] D. F. Anderson and P. S. Livingston, The zero-divisor graph of a commutative ring, J. Algebra 217 (1999), no. 2, 434-447.

[4] M. F. Atiyah and I. G. Macdonald, Introduction to Commutative Algebra, AddisonWesley, Readig, MA, 1969.

[5] B. Bollobás, Graph Theory: An Introduction Course, Springer-Verlag, New York, 1979.

[6] A. Yousefian Darani, Primal and weakly primal submodules and related results, Ph. D. thesis, University of Guilan, Iran, 2008.

7] L. Fuchs, On primal ideals, Proc. Amer. Math. Soc. 1 (1950), 1-6.

[8] S. P. Redmond, An ideal-based zero-divisor graph of a commutative ring, Comm. Algebra 31 (2003), no. 9, 4425-4443.

Ahmad ABbasi

Department of Pure Mathematics

Faculty of Mathematical Sciences

UNIVERSITY OF GUILAN

P. O. Box 41335-19141, RASHT, IRAN

E-mail address: aabbasi@guilan.ac.ir

SHOKOOFE HABIBI

Department of Pure Mathematics

Faculty of Mathematical Sciences

University of GuILAN

P. O. Box 41335-19141, RASHT, IRAN 